\title{
ON AN EQUATION RELATED TO NONADDITIVE ENTROPIES IN INFORMATION THEORY
}

\author{
PREM NATH AND DHIRAJ KUMAR SINGH*
}

\begin{abstract}
The paper provides the general solutions of a sum form functional equation containing three unknown mappings with some of the solutions related to the nonadditive entropies in information theory.
\end{abstract}

\section{INTRODUCTION}

For $n=1,2, \ldots$, let $\Gamma_{n}=\left\{\left(p_{1}, \ldots, p_{n}\right): p_{i} \geq 0, i=1, \ldots, n ; \sum_{i=1}^{n} p_{i}=1\right\}$ denote the set of all $n$-component finite discrete complete probability distributions with nonnegative elements.

Behara and Nath [1] considered the functional equation

$$
\sum_{i=1}^{n} \sum_{j=1}^{m} f\left(p_{i} q_{j}\right)=\sum_{i=1}^{n} p_{i}^{\alpha} \sum_{j=1}^{m} f\left(q_{j}\right)+\sum_{j=1}^{m} q_{j}^{\beta} \sum_{i=1}^{n} f\left(p_{i}\right)
$$

with $f: I \rightarrow \mathbb{R}$ an unknown mapping, $I=\{x \in \mathbb{R}: 0 \leq x \leq 1\}, \mathbb{R}$ denoting the set of all real numbers; $\left(p_{1}, \ldots, p_{n}\right) \in \Gamma_{n},\left(q_{1}, \ldots, q_{m}\right) \in \Gamma_{m} ; n=1,2, \ldots$; $m=1,2, \ldots ; \alpha$ and $\beta$ being fixed positive real powers; $0^{\alpha}:=0,0^{\beta}:=0$ and $1^{\alpha}:=1,1^{\beta}:=1$. They found the continuous solutions of (1.1).

Losonczi and Maksa ([3], p. 263) considered the functional equation (1.1) with $f: I \rightarrow \mathbb{R}$ an unknown mapping, $\left(p_{1}, \ldots, p_{n}\right) \in \Gamma_{n},\left(q_{1}, \ldots, q_{m}\right) \in \Gamma_{m} ; n \geq 3$ and $m \geq 2$ being fixed integers; $1 \neq \alpha \in \mathbb{R}, 1 \neq \beta \in \mathbb{R} ; 0^{\alpha}:=0,0^{\beta}:=0$; $1^{\alpha}:=1,1^{\beta}:=1$; and they found the general solutions of (1.1) without imposing any regularity condition on the mapping $f: I \rightarrow \mathbb{R}$.

The object of this paper is to determine the general solutions of the functional equation

$$
\sum_{i=1}^{n} \sum_{j=1}^{m} f\left(p_{i} q_{j}\right)=\sum_{i=1}^{n} g\left(p_{i}\right) \sum_{j=1}^{m} h\left(q_{j}\right)+\sum_{j=1}^{m} q_{j}^{\beta} \sum_{i=1}^{n} h\left(p_{i}\right)
$$

with $\left(p_{1}, \ldots, p_{n}\right) \in \Gamma_{n},\left(q_{1}, \ldots, q_{m}\right) \in \Gamma_{m} ; n \geq 3$ and $m \geq 3$ being fixed integers; $\beta$ a fixed positive real power satisfying the conventions $0^{\beta}:=0,1^{\beta}:=1 ; f, g, h$ being unknown real-valued mappings each with domain $I$.

$M S C$ (2010): primary 39B22; secondary 39B52.

Keywords: functional equation, additive mapping, multiplicative mapping, logarithmic mapping.

${ }^{\star}$ Corresponding author. 
If $g(x)=x$ and $\beta=1$, then (A) reduces to the equation

$$
\sum_{i=1}^{n} \sum_{j=1}^{m} f\left(p_{i} q_{j}\right)=\sum_{i=1}^{n} h\left(p_{i}\right)+\sum_{j=1}^{m} h\left(q_{j}\right)
$$

which has been discussed in [5].

If $g(x)=x^{\alpha}$ for all $x \in I, \alpha$ being a fixed positive real power; $0^{\alpha}:=0,1^{\alpha}:=1$, then $(\mathrm{A})$ reduces to the functional equation

$$
\sum_{i=1}^{n} \sum_{j=1}^{m} f\left(p_{i} q_{j}\right)=\sum_{i=1}^{n} p_{i}^{\alpha} \sum_{j=1}^{m} h\left(q_{j}\right)+\sum_{j=1}^{m} q_{j}^{\beta} \sum_{i=1}^{n} h\left(p_{i}\right)
$$

which is a Pexider-type generalization of (1.1). Notice that (1.2) reduces to (1.1) when $h(x)=f(x)$ for all $x \in I$. Some results concerning the functional equation (1.2) will be presented elsewhere.

Now, we mention below some definitions and results needed for the development of subsequent sections of this paper.

A mapping $a: \mathbb{R} \rightarrow \mathbb{R}$ is said to be additive if $a(x+y)=a(x)+a(y)$ for all $x \in \mathbb{R}, y \in \mathbb{R}$.

A mapping $M: I \rightarrow \mathbb{R}$ is said to be multiplicative if $M(x y)=M(x) M(y)$ for all $x \in I, y \in I$.

A mapping $\ell: I \rightarrow \mathbb{R}$ is said to be logarithmic if $\ell(0)=0$ and $\ell(x y)=\ell(x)+\ell(y)$ for all $x \in] 0,1], y \in] 0,1]] 0,1,]=\{x \in \mathbb{R}: 0<x \leq 1\}$.

Result 1.1 ([4]). Let a mapping $\phi: I \rightarrow \mathbb{R}$ satisfy the functional equation $\sum_{i=1}^{n} \phi\left(p_{i}\right)=c$ for all $\left(p_{1}, \ldots, p_{n}\right) \in \Gamma_{n}, n \geq 3$ a fixed integer and $c$ a given real constant. Then, there exists an additive mapping $A: \mathbb{R} \rightarrow \mathbb{R}$ such that $\phi(p)=$ $A(p)-\frac{1}{n} A(1)+\frac{c}{n}$ for all $p \in I$.

Result $1.2([6])$. Let $n \geq 3, m \geq 3$ be fixed integers. If mappings $H: I \rightarrow \mathbb{R}$, $G: I \rightarrow \mathbb{R}$ satisfy the equation

$$
\begin{aligned}
\sum_{i=1}^{n} \sum_{j=1}^{m} H\left(p_{i} q_{j}\right)= & \sum_{i=1}^{n} G\left(p_{i}\right) \sum_{j=1}^{m} H\left(q_{j}\right)+\sum_{i=1}^{n} H\left(p_{i}\right) \sum_{j=1}^{m} q_{j}^{\beta} \\
& +(m-n) H(0) \sum_{j=1}^{m} q_{j}^{\beta}+m(n-1) H(0)
\end{aligned}
$$

for all $\left(p_{1}, \ldots, p_{n}\right) \in \Gamma_{n},\left(q_{1}, \ldots, q_{m}\right) \in \Gamma_{m} ; \beta \neq 1$ a fixed positive real power such that $0^{\beta}:=0$ and $1^{\beta}:=1$. Then, any general solution of $(B)$ is of the form

$\left.\begin{array}{l}\text { (i) } H(p)=[H(1)+(m-1) H(0)] p^{\beta}+b_{1}(p)+H(0) ; b_{1}(1)=-m H(0) \\ \text { (ii) } G(p)=a_{1}(p)+G(0) ; a_{1}(1)=-n G(0)\end{array}\right\}$

or

$$
\left.\begin{array}{rl}
\text { (i) } H(p) & =p^{\beta} \ell(p)-\bar{b}(p)+H(0) ; \bar{b}(1)=m H(0) \\
\text { (ii) } G(p)=p^{\beta}+\bar{a}(p)+G(0) ; \bar{a}(1)=-n G(0)
\end{array}\right\}
$$


or

$$
\left.\begin{array}{l}
\text { (i) } H(p)=b_{2}(p)+H(0) ; b_{2}(1)=-m H(0) \\
\text { (ii) } \quad G \text { an arbitrary real-valued mapping }
\end{array}\right\}
$$

or

$$
\left.\begin{array}{rl}
\text { (i) } H(p) & =d\left[a(p)-p^{\beta}\right]+H(0) ; a(1)=1-\frac{m}{d} H(0) \\
\text { (ii) } \quad G(p)=a(p)+\bar{B}(p)+G(0) ; \bar{B}(1) & =-n G(0)+\frac{m}{d} H(0)
\end{array}\right\}
$$

or

(i) $H(p)=d\left[M(p)-b(p)-p^{\beta}\right]+H(0) ; b(1)=\frac{m}{d} H(0)$

(ii) $\left.G(p)=M(p)-b(p)+\bar{B}(p)+G(0) ; \bar{B}(1)=-n G(0)+\frac{m}{d} H(0)\right\}$

where $M: I \rightarrow \mathbb{R}$ is a nonadditive multiplicative mapping with $M(0)=0, M(1)=$ $1 ; \ell: I \rightarrow \mathbb{R}$ is a logarithmic mapping; $d$ is an arbitrary nonzero real constant; $a_{1}: \mathbb{R} \rightarrow \mathbb{R}, b_{i}: \mathbb{R} \rightarrow \mathbb{R}(i=1,2), \bar{a}: \mathbb{R} \rightarrow \mathbb{R}, \bar{b}: \mathbb{R} \rightarrow \mathbb{R}, a: \mathbb{R} \rightarrow \mathbb{R}, b: \mathbb{R} \rightarrow \mathbb{R}$ and $\bar{B}: \mathbb{R} \rightarrow \mathbb{R}$ are additive mappings.

Note. If $G(p)=p^{\alpha}, \alpha>0, \alpha \neq 1, p \in I$, then (B) reduces to the functional equation

$$
\begin{aligned}
\sum_{i=1}^{n} \sum_{j=1}^{m} H\left(p_{i} q_{j}\right)= & \sum_{i=1}^{n} p_{i}^{\alpha} \sum_{j=1}^{m} H\left(q_{j}\right)+\sum_{i=1}^{n} H\left(p_{i}\right) \sum_{j=1}^{m} q_{j}^{\beta} \\
& +(m-n) H(0) \sum_{j=1}^{m} q_{j}^{\beta}+m(n-1) H(0)
\end{aligned}
$$

which may be regarded as an enlargement of (1.1) (with $H$ in place of $f$ ). Its solutions will be presented elsewhere.

\section{The MAIn RESUlT}

The main result of this paper is the following:

Theorem 2.1. Let $n \geq 3, m \geq 3$ be fixed integers and $f: I \rightarrow \mathbb{R}, g: I \rightarrow \mathbb{R}, h$ : $I \rightarrow \mathbb{R}$ be mappings which satisfy the functional equation $(A)$ for all $\left(p_{1}, \ldots, p_{n}\right) \in$ $\Gamma_{n},\left(q_{1}, \ldots, q_{m}\right) \in \Gamma_{m} ; \beta \neq 1$ being a fixed positive real power such that $0^{\beta}:=0$, $1^{\beta}:=1$. Then, any general solution $(f, g, h)$ of $(A)$ is of the form

$$
\begin{aligned}
& \text { (i) } f(p)=[h(1)+(n-1) h(0)] p^{\beta}+A_{1}(p)+f(0) \\
& \text { (ii) } g(p)=A_{2}(p)+g(0) \\
& \text { (iii) } h(p)=[h(1)+(n-1) h(0)] p^{\beta}+\bar{A}(p)+h(0)
\end{aligned}
$$

or

(i) $\quad f(p)=[g(1)+(n-1) g(0)]\left\{[h(1)+(m-1) h(0)] p^{\beta}+A_{3}(p)\right\}$

$$
+[h(1)+(n-1) h(0)] p^{\beta}+A_{1}(p)+f(0)
$$

(ii) $g$ an arbitrary real-valued mapping

(iii) $h(p)=[h(1)+(m-1) h(0)] p^{\beta}+A_{3}(p)+h(0)$ 
or
(i) $\quad f(p)=\{[g(1)+(n-1) g(0)] \ell(p)+[f(1)+(n m-1) f(0)]\} p^{\beta}$ $-[g(1)+(n-1) g(0)] \bar{b}(p)+A_{1}(p)+f(0)$
(ii) $g(p)=[g(1)+(n-1) g(0)]\left[p^{\beta}+\bar{a}(p)\right]+g(0)$
(iii) $h(p)=\{\ell(p)+[h(1)+(m-1) h(0)]\} p^{\beta}-\bar{b}(p)+h(0)$

with $[g(1)+(n-1) g(0)] \neq 0$ or
(i) $\quad f(p)=\{[f(1)+(n m-1) f(0)]-d[g(1)+(n-1) g(0)]\} p^{\beta}$ $+d[g(1)+(n-1) g(0)] a(p)+A_{1}(p)+f(0)$
(ii) $g(p)=[g(1)+(n-1) g(0)][a(p)+\bar{B}(p)]+g(0)$
(iii) $h(p)=\{[h(1)+(m-1) h(0)]-d\} p^{\beta}+d a(p)+h(0)$

with $[g(1)+(n-1) g(0)] \neq 0$ or
(i) $\quad f(p)=d[g(1)+(n-1) g(0)][M(p)-b(p)]+\{[f(1)+(n m-1) f(0)]$ $-d[g(1)+(n-1) g(0)]\} p^{\beta}+A_{1}(p)+f(0)$
(ii) $g(p)=[g(1)+(n-1) g(0)][M(p)-b(p)+\bar{B}(p)]+g(0)$
(iii) $h(p)=d[M(p)-b(p)]+\{[h(1)+(m-1) h(0)]-d\} p^{\beta}+h(0)$

with $[g(1)+(n-1) g(0)] \neq 0$. Moreover,

(i) $[g(1)+(n-1) g(0)-1][h(p)-h(0)]$

$$
=[h(1)+(m-1) h(0)][g(p)-g(0)]-[h(1)+(n-1) h(0)] p^{\beta}+B(p)
$$

(ii) $f(1)+(n m-1) f(0)=[g(1)+(n-1) g(0)][h(1)+(m-1) h(0)]$

$$
+[h(1)+(n-1) h(0)]
$$

$M: I \rightarrow \mathbb{R}$ is a nonadditive multiplicative mapping with $M(0)=0$ and $M(1)=1$; $\ell: I \rightarrow \mathbb{R}$ is a logarithmic mapping; $d \neq 0$ is an arbitrary real constant; $A_{i}: \mathbb{R} \rightarrow \mathbb{R}$ $(i=1,2,3), \bar{b}: \mathbb{R} \rightarrow \mathbb{R}, \bar{a}: \mathbb{R} \rightarrow \mathbb{R}, a: \mathbb{R} \rightarrow \mathbb{R}, b: \mathbb{R} \rightarrow \mathbb{R}, \bar{A}: \mathbb{R} \rightarrow \mathbb{R}, B: \mathbb{R} \rightarrow \mathbb{R}$, 
$\bar{B}: \mathbb{R} \rightarrow \mathbb{R}$ are additive mappings such that

$$
\begin{aligned}
\text { (i) } & A_{1}(1)=-n m f(0)+m[g(1)+(n-1) g(0)] h(0) \\
\text { (ii) } & A_{2}(1)=-n g(0) \\
\text { (iii) } & A_{3}(1)=-m h(0) \\
\text { (iv) } & a(1)=1-\frac{m}{d} h(0) \\
\text { (v) } & b(1)=\frac{m}{d} h(0) \\
\text { (vi) } & \bar{a}(1)=-n[g(1)+(n-1) g(0)]^{-1} g(0) \\
\text { (vii) } & \bar{b}(1)=m h(0) \\
\text { (viii) } & \bar{B}(1)=-n[g(1)+(n-1) g(0)]^{-1} g(0)+\frac{m}{d} h(0) \\
\text { (ix) } & B(1)=n h(0)+n[h(1)+(m-1) h(0)] g(0) \\
& \quad-m[g(1)+(n-1) g(0)] h(0) \\
\text { (x) } & \bar{A}(1)=-n h(0) .
\end{aligned}
$$

Proof. Putting $p_{1}=1, p_{2}=\ldots=p_{n}=0$ and $q_{1}=1, q_{2}=\ldots=q_{m}=0$ in (A), $(\gamma)$ (ii) follows. Now, let us put $p_{1}=1, p_{2}=\ldots=p_{n}=0$ in (A). We obtain

$$
\sum_{j=1}^{m}\left\{f\left(q_{j}\right)-[g(1)+(n-1) g(0)] h\left(q_{j}\right)-[h(1)+(n-1) h(0)] q_{j}^{\beta}\right\}=-m(n-1) f(0) .
$$

By Result 1.1, there exists an additive mapping $A_{1}: \mathbb{R} \rightarrow \mathbb{R}$ such that

$$
f(p)=[g(1)+(n-1) g(0)][h(p)-h(0)]+[h(1)+(n-1) h(0)] p^{\beta}+A_{1}(p)+f(0)
$$

with $A_{1}(1)$ given by $(\delta)(\mathrm{i})$. From equations $(\mathrm{A})$ and $(2.1)$, it follows that

$$
\begin{aligned}
& {[g(1)+(n-1) g(0)] \sum_{i=1}^{n} \sum_{j=1}^{m} h\left(p_{i} q_{j}\right)+[h(1)+(n-1) h(0)] \sum_{i=1}^{n} \sum_{j=1}^{m}\left(p_{i} q_{j}\right)^{\beta}} \\
& =\sum_{i=1}^{n} g\left(p_{i}\right) \sum_{j=1}^{m} h\left(q_{j}\right)+\sum_{i=1}^{n} h\left(p_{i}\right) \sum_{j=1}^{m} q_{j}^{\beta}+m(n-1)[g(1)+(n-1) g(0)] h(0) .
\end{aligned}
$$

The substitutions $q_{1}=1, q_{2}=\ldots=q_{m}=0$ in $(2.2)$ give

$$
\begin{aligned}
\sum_{i=1}^{n} h\left(p_{i}\right) & =[g(1)+(n-1) g(0)] \sum_{i=1}^{n} h\left(p_{i}\right)-[h(1)+(m-1) h(0)] \sum_{i=1}^{n} g\left(p_{i}\right) \\
& +[h(1)+(n-1) h(0)] \sum_{i=1}^{n} p_{i}^{\beta}+(m-n)[g(1)+(n-1) g(0)] h(0) .
\end{aligned}
$$

Let us write (2.3) in the form

$$
\begin{aligned}
\sum_{i=1}^{n}\{ & {[g(1)+(n-1) g(0)-1] h\left(p_{i}\right)-[h(1)+(m-1) h(0)] g\left(p_{i}\right) } \\
& \left.+[h(1)+(n-1) h(0)] p_{i}^{\beta}\right\}=-(m-n)[g(1)+(n-1) g(0)] h(0) .
\end{aligned}
$$


By Result 1.1, there exists an additive mapping $B: \mathbb{R} \rightarrow \mathbb{R}$ such that $(\gamma)$ (i) holds with $B(1)$ given by $(\delta)(\mathrm{ix})$.

From equations (2.2) and (2.3), we obtain

$$
\begin{aligned}
{[g(1)+(n-1) g(0)] } & \sum_{i=1}^{n} \sum_{j=1}^{m} h\left(p_{i} q_{j}\right) \\
= & \sum_{i=1}^{n} g\left(p_{i}\right) \sum_{j=1}^{m}\left\{h\left(q_{j}\right)-[h(1)+(m-1) h(0)] q_{j}^{\beta}\right\} \\
+[g(1)+(n-1) g(0)] & \left\{\sum_{i=1}^{n} h\left(p_{i}\right) \sum_{j=1}^{m} q_{j}^{\beta}+(m-n) h(0) \sum_{j=1}^{m} q_{j}^{\beta}+m(n-1) h(0)\right\} .
\end{aligned}
$$

Case 1. $g(1)+(n-1) g(0)=0$.

In this case, equation (2.4) reduces to the equation

$$
\sum_{i=1}^{n} g\left(p_{i}\right) \sum_{j=1}^{m}\left\{h\left(q_{j}\right)-[h(1)+(m-1) h(0)] q_{j}^{\beta}\right\}=0
$$

valid for all $\left(p_{1}, \ldots, p_{n}\right) \in \Gamma_{n},\left(q_{1}, \ldots, q_{m}\right) \in \Gamma_{m} ; n \geq 3, m \geq 3$ being fixed integers.

Consider the situation of $\sum_{i=1}^{n} g\left(p_{i}\right)=0$ for all $\left(p_{1}, \ldots, p_{n}\right) \in \Gamma_{n}$. By Result 1.1, there exists an additive mapping $A_{2}: \mathbb{R} \rightarrow \mathbb{R}$ such that $\left(\alpha_{1}\right)$ (ii) follows with $A_{2}(1)$ given by $(\delta)(\mathrm{ii})$. Also, from $\left(\alpha_{1}\right)(\mathrm{ii})$ and $(2.1) ;\left(\alpha_{1}\right)(\mathrm{i})$ follows with $A_{1}(1)=$ $-n m f(0)$ which can be obtained from $(\delta)(i)$ when $g(1)+(n-1) g(0)=0$. Now, let us put $q_{1}=1, q_{2}=\ldots=q_{m}=0$ in (A) and use $\left(\alpha_{1}\right)(\mathrm{i}),\left(\alpha_{1}\right)$ (ii). We obtain $\sum_{i=1}^{n}\left\{h\left(p_{i}\right)-[h(1)+(n-1) h(0)] p_{i}^{\beta}\right\}=0$. By Result 1.1, there exists an additive mapping $\bar{A}: \mathbb{R} \rightarrow \mathbb{R}$ such that $\left(\alpha_{1}\right)$ (iii) holds with $\bar{A}(1)$ given by $(\delta)(\mathrm{x})$. So, the solution $\left(\alpha_{1}\right)$ has been obtained with $A_{1}(1)=-n m f(0)$. Now, consider the situation of $\sum_{j=1}^{m}\left\{h\left(q_{j}\right)-[h(1)+(m-1) h(0)] q_{j}^{\beta}\right\}=0 \quad$ for all $\left(q_{1}, \ldots, q_{m}\right) \in \Gamma_{m}$. Making use of Result 1.1, it follows that there exists an additive mapping $A_{3}$ : $\mathbb{R} \rightarrow \mathbb{R}$ with $A_{3}(1)$ given by $(\delta)$ (iii), such that $\left(\alpha_{2}\right)$ (iii) holds. Now, from $\left(\alpha_{2}\right)$ (iii) and (2.5), one can easily conclude that $g$ is an arbitrary real-valued mapping with $g(1)+(n-1) g(0)=0$. Also, from $(2.1)$, it follows that $f(p)=[h(1)+(n-$ 1) $h(0)] p^{\beta}+A_{1}(p)+f(0)$ with $A_{1}(1)=-n m f(0)$. This solution is included in $\left(\alpha_{2}\right)$.

Case 2. $g(1)+(n-1) g(0) \neq 0$.

In this case, let us write (2.4) in the form

$$
\begin{aligned}
& \sum_{i=1}^{n} \sum_{j=1}^{m}\left\{h\left(p_{i} q_{j}\right)-[h(1)+(m-1) h(0)]\left(p_{i} q_{j}\right)^{\beta}\right\} \\
& \quad=\sum_{i=1}^{n}\left\{[g(1)+(n-1) g(0)]^{-1} g\left(p_{i}\right)\right\} \sum_{j=1}^{m}\left\{h\left(q_{j}\right)-[h(1)+(m-1) h(0)] q_{j}^{\beta}\right\}
\end{aligned}
$$




$$
\begin{aligned}
& +\sum_{i=1}^{n}\left\{h\left(p_{i}\right)-[h(1)+(m-1) h(0)] p_{i}^{\beta}\right\} \sum_{j=1}^{m} q_{j}^{\beta} \\
& +(m-n) h(0) \sum_{j=1}^{m} q_{j}^{\beta}+m(n-1) h(0) .
\end{aligned}
$$

Define the mappings $G: I \rightarrow \mathbb{R}$ and $H: I \rightarrow \mathbb{R}$ as

$$
G(p)=[g(1)+(n-1) g(0)]^{-1} g(p)
$$

and

$$
H(p)=h(p)-[h(1)+(m-1) h(0)] p^{\beta}
$$

for all $p \in I$. From (2.7) and (2.8), it is easy to conclude that

$$
\begin{aligned}
& G(0)=\frac{g(0)}{g(1)+(n-1) g(0)}, \quad g(1)+(n-1) g(0) \neq 0 \\
& G(1)+(n-1) G(0)=1 \\
& H(0)=h(0)
\end{aligned}
$$

and

$$
H(1)+(m-1) H(0)=0 .
$$

Also, from (2.6), (2.7) and (2.8), equation (B) follows. Therefore, in Result 1.2, we have to consider only those solutions which satisfy both (2.10) and (2.12). We reject $\left(\beta_{1}\right)$ because, in this case, $(2.10)$ does not hold. Both (2.10) and (2.12) hold in $\left(\beta_{2}\right),\left(\beta_{4}\right)$ and $\left(\beta_{5}\right)$. In $\left(\beta_{3}\right),(2.12)$ holds but $\left(\beta_{3}\right)$ can be accepted provided we consider only those arbitrary mappings $G$ which also satisfy (2.10). This requirement is met by any arbitrary mapping $g$ which satisfies the condition $[g(1)+(n-1) g(0)] \neq 0$. This fact is obvious from $(2.7)$. Keeping in view these observations, $\left(\alpha_{2}\right)$ with $[g(1)+(n-1) g(0)] \neq 0$ and $(\delta)\left((\mathrm{i})\right.$, (iii)) follows from $\left(\beta_{3}\right)$, (2.7), (2.8), (2.9), (2.11) and (2.1). Moreover,

$\left(a_{1}\right)$ The solution $\left(\alpha_{3}\right)$ with $[g(1)+(n-1) g(0)] \neq 0$ and $(\gamma)(\mathrm{ii}),(\delta)((\mathrm{i}),(\mathrm{vi})$, (vii)) follows from $\left(\beta_{2}\right),(2.7),(2.8),(2.9),(2.11)$ and $(2.1)$;

$\left(a_{2}\right)$ The solution $\left(\alpha_{4}\right)$ with $[g(1)+(n-1) g(0)] \neq 0$ and $(\gamma)(\mathrm{ii}),(\delta)((\mathrm{i}),(\mathrm{iv})$, (viii)) follows from $\left(\beta_{4}\right),(2.7),(2.8),(2.9),(2.11)$ and $(2.1)$;

$\left(a_{3}\right)$ The solution $\left(\alpha_{5}\right)$ with $[g(1)+(n-1) g(0)] \neq 0$ and $(\gamma)(\mathrm{ii}),(\delta)((\mathrm{i}),(\mathrm{v})$, (viii)) follows from $\left(\beta_{5}\right),(2.7),(2.8),(2.9),(2.11)$ and $(2.1)$.

Making use of $(\gamma)\left((\mathrm{i})\right.$, (ii)), it can be verified that $\left(\alpha_{1}\right)$ to $\left(\alpha_{5}\right)$ are, indeed, the solutions of $(\mathrm{A})$.

\section{Comments}

The object of this section is to comment upon various solutions, mentioned in Theorem 2.1, from the point of view of information theory. 
Behara and Nath [1] have defined the entropy $H_{n}^{(\alpha, \beta)}\left(p_{1}, \ldots, p_{n}\right)$ of type $(\alpha, \beta)$ $\left(H_{n}^{(\alpha, \beta)}: \Gamma_{n} \rightarrow \mathbb{R}, n=1,2, \ldots\right)$ as

$$
H_{n}^{(\alpha, \beta)}\left(p_{1}, \ldots, p_{n}\right)= \begin{cases}\left(2^{1-\alpha}-2^{1-\beta)}\right)^{-1}\left(\sum_{i=1}^{n} p_{i}^{\alpha}-\sum_{i=1}^{n} p_{i}^{\beta}\right) & \text { if } \alpha \neq \beta \\ -2^{\beta-1} \sum_{i=1}^{n} p_{i}^{\beta} \log _{2} p_{i} & \text { if } \alpha=\beta\end{cases}
$$

where $0^{\beta} \log _{2} 0:=0$ and $\alpha, \beta$ are fixed positive real powers which satisfy $0^{\alpha}:=0$, $0^{\beta}:=0,1^{\alpha}:=1,1^{\beta}:=1$. Havrda and Charvát [2] have defined the entropies of degree $\beta, 0<\beta \in \mathbb{R}, \beta \neq 1$ as

$$
H_{n}^{\beta}\left(p_{1}, \ldots, p_{n}\right)=\left(1-2^{1-\beta}\right)^{-1}\left[1-\sum_{i=1}^{n} p_{i}^{\beta}\right]
$$

with $H_{n}^{\beta}: \Gamma_{n} \rightarrow \mathbb{R}, n=1,2, \ldots$ and $0^{\beta}:=0,1^{\beta}:=1$. Both the entropies mentioned in (3.1) and (3.2) are nonadditive.

I. In the solution $\left(\alpha_{1}\right), \sum_{i=1}^{n} g\left(p_{i}\right)=0$ follows. Then, equation (A) reduces to

$$
\sum_{i=1}^{n} \sum_{j=1}^{m} f\left(p_{i} q_{j}\right)=\sum_{j=1}^{m} q_{j}^{\beta} \sum_{i=1}^{n} h\left(p_{i}\right) .
$$

The functional equation (3.3) has been discussed by Nath and Singh [6].

II. In the solution $\left(\alpha_{2}\right)$, the mapping $g$ is arbitrary. So, there are three possibilities: $g$ is additive or $g$ is multiplicative or $g$ is logarithmic.

Let us consider the case of $g$ being additive. If $g(p) \equiv 0$, then $g$ is certainly additive. In this case, (A) reduces to (3.3). In general, when $g$ is additive, (A) reduces to

$$
\sum_{i=1}^{n} \sum_{j=1}^{m} f\left(p_{i} q_{j}\right)=g(1) \sum_{j=1}^{m} h\left(q_{j}\right)+\sum_{j=1}^{m} q_{j}^{\beta} \sum_{i=1}^{n} h\left(p_{i}\right) .
$$

If $g(1)=0$, we get the functional equation (3.3) again. If $g(1)=1$, then (3.4) reduces to the equation

$$
\sum_{i=1}^{n} \sum_{j=1}^{m} f\left(p_{i} q_{j}\right)=\sum_{j=1}^{m} h\left(q_{j}\right)+\sum_{j=1}^{m} q_{j}^{\beta} \sum_{i=1}^{n} h\left(p_{i}\right) .
$$

Consider the case of $\mathrm{g}$ being multiplicative. Here, we discuss some particular cases.

(i) $g(x) \equiv 0$. In this case, we again get (3.3)

(ii) $g(x) \equiv 1$. In this case, (A) reduces to

$$
\sum_{i=1}^{n} \sum_{j=1}^{m} f\left(p_{i} q_{j}\right)=n \sum_{j=1}^{m} h\left(q_{j}\right)+\sum_{j=1}^{m} q_{j}^{\beta} \sum_{i=1}^{n} h\left(p_{i}\right)
$$

where $n=1,2$ are not admissible because we assume $n \geq 3$ throughout the paper.

(iii) $g(x)=x$ for all $x \in I$. In this case, equation (A) reduces to (3.5). 
(iv) $g(p)=\left\{\begin{array}{ll}p^{\delta} & \text { if } 0<p \leq 1 \\ 0 & \text { if } p=0\end{array}\right.$ where $\delta \in \mathbb{R}$. Some particular values of $\delta$ seem to be of interest from the point of view of applications. If $\delta=1$, then $g(p)=p$, which has already been discussed above. If $\delta=\alpha, \alpha>0, \alpha \neq 1$, then (A) reduces to (1.2). If $\delta=\beta, \beta>0, \beta \neq 1$, then (A) reduces to

$$
\sum_{i=1}^{n} \sum_{j=1}^{m} f\left(p_{i} q_{j}\right)=\sum_{i=1}^{n} p_{i}^{\beta} \sum_{j=1}^{m} h\left(q_{j}\right)+\sum_{j=1}^{m} q_{j}^{\beta} \sum_{i=1}^{n} h\left(p_{i}\right) .
$$

If $\delta=0$, then $g(p)$ can be written as $g(p)=\left\{\begin{array}{ll}1 & \text { if } 0<p \leq 1 \\ 0 & \text { if } p=0\end{array}\right.$. Therefore, equation $(\mathrm{A})$ reduces to

$$
\sum_{i=1}^{n} \sum_{j=1}^{m} g\left(p_{i} q_{j}\right)=N(P) \sum_{j=1}^{m} h\left(q_{j}\right)+\sum_{j=1}^{m} q_{j}^{\beta} \sum_{i=1}^{n} h\left(p_{i}\right)
$$

where $N(P)$ denotes the number of non-zero elements in the probability distribution $P=\left(p_{1}, \ldots, p_{n}\right) \in \Gamma_{n}$.

(v) Consider the situation $g(p)=\left\{\begin{array}{ll}0 & \text { if } 0 \leq p<1 \\ 1 & \text { if } p=1\end{array}\right.$. In this case, $\sum_{i=1}^{n} g\left(p_{i}\right)=1$ if $p_{i}=1$ for exactly one $i, 1 \leq i \leq n$ and 0 if $0 \leq p_{i}<1, i=1,2, \ldots, n$.

Finally, we consider the case of $g(p)=\ell(p)$ where $\ell$ is a logarithmic mapping. If $\ell(p) \equiv 0$, then $(\mathrm{A})$ reduces to $(3.3)$.

Notice that, if $g(p) \equiv 0$, then $g$ is additive, multiplicative and logarithmic. In each case, the functional equation (3.3) arises. Such a discussion concerning $g(p) \equiv 0$ is needed for the sake of completeness in relation to solution $\left(\alpha_{2}\right)$.

III. From the solution $\left(\alpha_{3}\right)$, it can be easily derived that for all $\left(p_{1}, \ldots, p_{n}\right) \in$ $\Gamma_{n}$

$$
\begin{aligned}
\sum_{i=1}^{n} f\left(p_{i}\right)= & {[g(1)+(n-1) g(0)] \sum_{i=1}^{n} p_{i}^{\beta} \ell\left(p_{i}\right)+[f(1)+(n m-1) f(0)] \sum_{i=1}^{n} p_{i}^{\beta} } \\
& -n(m-1) f(0) ; \\
\sum_{i=1}^{n} g\left(p_{i}\right)= & {[g(1)+(n-1) g(0)] \sum_{i=1}^{n} p_{i}^{\beta} ; } \\
\sum_{i=1}^{n} h\left(p_{i}\right)= & \sum_{i=1}^{n} p_{i}^{\beta} \ell\left(p_{i}\right)+[h(1)+(m-1) h(0)] \sum_{i=1}^{n} p_{i}^{\beta}+(n-m) h(0) .
\end{aligned}
$$

Let us choose the logarithmic mapping $\ell: I \rightarrow \mathbb{R}$ as

$$
\ell(p)= \begin{cases}\lambda \log _{2} p & \text { if } 0<p \leq 1 \\ 0 & \text { if } p=0\end{cases}
$$

where $\lambda \neq 0$ is an arbitrary real constant. Then, making use of (3.1), (3.2) and (3.6), we have

$$
\sum_{i=1}^{n} f\left(p_{i}\right)=-[g(1)+(n-1) g(0)] \lambda 2^{1-\beta} H_{n}^{(\beta, \beta)}\left(p_{1}, \ldots, p_{n}\right)
$$




$$
\begin{aligned}
& +[f(1)+(n m-1) f(0)]\left[-\left(1-2^{1-\beta}\right) H_{n}^{\beta}\left(p_{1}, \ldots, p_{n}\right)+1\right] \\
& -n(m-1) f(0) ; \\
\sum_{i=1}^{n} g\left(p_{i}\right)= & {[g(1)+(n-1) g(0)]\left[-\left(1-2^{1-\beta}\right) H_{n}^{\beta}\left(p_{1}, \ldots, p_{n}\right)+1\right] } \\
\sum_{i=1}^{n} h\left(p_{i}\right)= & -\lambda 2^{1-\beta} H_{n}^{(\beta, \beta)}\left(p_{1}, \ldots, p_{n}\right)+[h(1)+(m-1) h(0)] \\
& \times\left[-\left(1-2^{1-\beta}\right) H_{n}^{\beta}\left(p_{1}, \ldots, p_{n}\right)+1\right]+(n-m) h(0) .
\end{aligned}
$$

Thus, we observe that the mappings $f$ and $h$ are related to both the entropies $H_{n}^{(\beta, \beta)}$ and $H_{n}^{\beta}$ whereas the mapping $g$ is related only to the entropies $H_{n}^{\beta}$.

IV. From the solution $\left(\alpha_{4}\right)$, for all $\left(p_{1}, \ldots, p_{n}\right) \in \Gamma_{n}$, it can be derived that

$$
\begin{aligned}
\sum_{i=1}^{n} f\left(p_{i}\right)= & \{[f(1)+(n m-1) f(0)]-d[g(1)+(n-1) g(0)]\} \\
\times & {\left[-\left(1-2^{1-\beta}\right) H_{n}^{\beta}\left(p_{1}, \ldots, p_{n}\right)+1\right]+d[g(1)+(n-1) g(0)] } \\
& -n(m-1) f(0) ; \\
\sum_{i=1}^{n} g\left(p_{i}\right)= & {[g(1)+(n-1) g(0)] } \\
\sum_{i=1}^{n} h\left(p_{i}\right)= & \{[h(1)+(m-1) h(0)]-d\}\left[-\left(1-2^{1-\beta}\right) H_{n}^{\beta}\left(p_{1}, \ldots, p_{n}\right)+1\right] \\
& +d+(n-m) h(0) .
\end{aligned}
$$

Thus, we observe that the mappings $f$ and $h$ are related to entropies $H_{n}^{\beta}$ whereas the mapping $g$ is not related to any of the entropies given by (3.1) and (3.2).

V. If we choose a mapping $M: I \rightarrow \mathbb{R}$ defined as $M(p)=p^{\alpha}, p \in I, \alpha \in \mathbb{R}$, $\alpha>0, \alpha \neq 1, \alpha \neq \beta$, then, from solution $\left(\alpha_{5}\right)$, we obtain

$$
\begin{aligned}
\sum_{j=1}^{m} f\left(q_{j}\right) & =d[g(1)+(n-1) g(0)]\left(2^{1-\alpha}-2^{1-\beta}\right) H_{m}^{(\alpha, \beta)}\left(q_{1}, \ldots, q_{m}\right) \\
& -[f(1)+(n m-1) f(0)]\left(1-2^{1-\beta}\right) H_{m}^{\beta}\left(q_{1}, \ldots, q_{m}\right) \\
& +[f(1)+(m-1) f(0)] ; \\
\sum_{j=1}^{m} g\left(q_{j}\right) & =[g(1)+(n-1) g(0)]\left[-\left(1-2^{1-\alpha}\right) H_{m}^{\alpha}\left(q_{1}, \ldots, q_{m}\right)+1\right]+(m-n) g(0) ; \\
\sum_{j=1}^{m} h\left(q_{j}\right) & =d\left(2^{1-\alpha}-2^{1-\beta}\right) H_{m}^{(\alpha, \beta)}\left(q_{1}, \ldots, q_{m}\right) \\
& -[h(1)+(m-1) h(0)]\left[\left(1-2^{1-\beta}\right) H_{m}^{\beta}\left(q_{1}, \ldots, q_{m}\right)-1\right] .
\end{aligned}
$$

Here, observe that the mappings in the solution $\left(\alpha_{5}\right)$ are related to the entropies of type $(\alpha, \beta)$ when $\alpha \neq \beta$; the entropies of degree $\alpha$ and the entropies of degree $\beta$. 
Acknowledgement. The authors are grateful to the referee for his/her valuable suggestions.

\section{REFERENCES}

[1] M. Behara and P. Nath, Information and entropy of countable measurable partitions I, Kybernetika 10 (1974), 491-503.

[2] J. Havrda and F. Charvát, Quantification method of classification process. Concept of structural $\alpha$-entropy, Kybernetika 3 (1967), 30-35.

[3] L. Losonczi and Gy. Maksa, The general solution of a functional equation of information theory, Glas. Mat., III. Ser. 16 (1981), 261-266.

[4] L. Losonczi and Gy. Maksa, On some functional equations of the information theory, Acta Math. Acad. Sci. Hung. 39 (1982), 73-82.

[5] P. Nath and D. K. Singh, The general solutions of a functional equation related to information theory, Ratio Math. 15 (2005), 1-24.

[6] P. Nath and D. K. Singh, On a sum form functional equation related to various nonadditive entropies in information theory, Tamsui Oxf. J. Inf. Math. Sci. 30 (2014), 23-43.

Prem Nath, Department of Mathematics, University of Delhi, Delhi-110007, India e-mail: pnathmaths@gmail.com

Dhiraj Kumar Singh, Department of Mathematics, Zakir Husain Delhi College (University of Delhi), Jawaharlal Nehru Marg, Delhi-110002, India

e-mail: dhiraj426@rediffmail.com, dksingh@zh.du.ac.in 
